\title{
Knowledge and perceptions of antenatal services need and delivery and reasons for seeking such services among women in Tanzania: Implications for utilization and coverage of intermittent presumptive treatment of malaria in pregnancy in two districts
}

\author{
Godfrey M. Mubyazi* \\ National Institute for Medical Research P.O. Box 9653 Dar es Salaam, Tanzania.
}

\begin{abstract}
Background: Learning from a volume of the literature that lacks information regarding people's opportunity to access health services for disease control, a study was conducted to assess knowledge, perceptions of antenatal care (ANC) services and actually delivered services and reasons for seeking ANC including intermittent presumptive treatment during pregnancy (IPTp) against malaria in Mkuranga and Mufindi districts, Tanzania. Methodology: Quantitative and qualitative techniques were employed, involving interviews with ANC clients, informal communications with health care workers, focus group discussions with mothers of young children, and intertemporal observations. Data were collected for six months in 2006. Results: ANC seeking was mainly influenced by motivation for one's safe pregnancy and childbirth and not necessarily and specifically seeking IPTp. The main barriers reported to hinder pregnant women effectively utilize ANC include socio-cultural values stigmatizing and discriminating women during pregnancy, service providers' unfriendly attitudes towards clients, shortages of drugs and other essential service supplies, existence of user-fees in health facilities, and pregnant women's unawareness of essential ANC services needed or actually delivered at ANC clinics. Conclusion: While client demand for services depends on volume, cost/price and quality of services supplied, lack of knowledge of what one deserves also influences one's care seeking behavior. Therefore, it is imperative to do periodic evaluations of population knowledge, perceptions of the introduced policy, and service delivery programs if the health care system is to be made more effective by providing a room for corrective actions.
\end{abstract}

Key words: antenatal care, malaria, pregnancy, maternal health, intermittent preventive treatment

\section{Background}

The association between health seeking behavior and health care utilization is a complex phenomenon as literature widely supports. However, immense evidence from systematic studies on demonstrates an influence of various factors on health service use in different communities. It is revealed that knowledge, beliefs and attitudes in relation to disease and health services are among the key determinants of health service utilization (Chakraborthy et al., 2003; Haussman-Muela et al., 2003). Meanwhile, theorists in the field of social sciences and humanities argue that knowledge and beliefs about health problems and the methods for the control of such problems may be distinguished from each other. They depict that while the two elements are not synonymous, beliefs may sometimes be influenced by one's knowledge, and both of them or the association between them may have implications for a person's or society's health seeking behavior (Mubyazi 2010). While experts tend to argue that a person may have knowledge that is not always translated into practice (Hausmann-Muela et al., 2003), and that knowledge alone may not influence people's behaviors when it comes to utilization of specific health services or interventions (Nganda et al., 2004), others maintain that knowledge about health enables people to recognize, respect and properly exercise their health rights and to maintain their wellbeing (Maputle et al., 2013).

In the area of maternal and child health $(\mathrm{MCH})$ the need for understanding the status of knowledge of both the service providers and targeted service users, their perceptions of the service/intervention being promoted, as well as general populations or specific group's health seeking behavior is remains urgent. This would help the authorities concerned to identify the determinants of health seeking behavior in relation to particular health services/interventions and design mechanisms that would promote the provision and coverage of specific services/ interventions towards attainment of the predetermined goal (Maputle et al., 2013).

In the area of antenatal care (ANC) services, medical and nursing experts suggest the need for pregnant women to seek the care needed while they are properly informed so that they can take appropriate actions in relation to the

*Correspondence: gmmubyazi@gmail.com; gmmubyazi@yahoo.co.uk 
care needed. It is recommended that the health service client needs to have understood both the information provided and the full implications of all the alternative courses of action available. For this reason, health service clients, for example, pregnant women in this case cannot make right ANC seeking and service use decisions if they lack right information at the right time and in a comprehensible way (National Collaborating Center for Women and Children 2008).

In the absence of the right and adequate knowledge about a particular health problem or service, people may develop perceptions or beliefs about such services/ problems in the wrong way. As literature establishes, this has been noted in the many areas of public health including the area of malaria-related services delivered in ANC clinic settings, therefore, contributing to limit the usability of such services in the respective community settings (Mubyazi et al., 2008a).

Intermittent presumptive/preventive treatment during pregnancy (IPTp) against malaria using sulfadoxinepyrimethamine (SP) is recommended by the World Health Organization (WHO) for countries facing stable or unstable malaria transmission epidemiology. ANC clinics are found to be the best places for delivering this novel intervention (WHO 2013). The government of Tanzania recommended officially all ANC clinics to adopt the WHO guideline for IPTp in 2001 (MoHSW 2004). The IPTp guideline requires each pregnant woman visiting an ANC and eligible to receive IPTp doses to do so by taking SP in the specified pregnancy gestational ages. At least two doses of SP for IPTp are recommended throughout a woman's pregnancy. The first dose should be taken during the second trimester immediately after quickening while the second dose should be taken during the third trimester. A one-month interval is recommended between the one dose and the next one. These doses are supposed to be administered by a qualified health worker by follow a direct observation therapy (DOT) guideline (WHO 2013). The national IPTp guidelines emphasizes that the clients eligible to receive IPTp doses should be given health educational messages about the use and benefits of SP (MoHSW 2004). However, there is still shortage of empirical evidence based on rigorous studies to inform policy on issues relating to community 9and in particular pregnant women's) knowledge on ANC service and its implications on the accessibility and utilization of IPTp-SP in SSA (Pell et al., 2011; Ribera et al., 2007; Nganda et al., 2004).

In Tanzania, the need for further studies on knowledge related determinants of ANC use including use of IPTp against malaria was identified nearly a decade ago
(Mubyazi et al., 2005; Mubyazi 2010). This paper is about a study that was conducted in two districts with the aim of describing ANC and malaria health literacy and reasons for seeking or avoiding seeking ANC among currently and recently pregnant women in two rural districts of Tanzania to inform improvements to ANC service and other maternal health delivery in rural Tanzania and similar settings.

\section{Methods}

Study design, areas, population and sampling approaches

The study from which the data from the presently reported and discussed data were drawn was a crosssectional survey by design, having adopted mixed data collection and analysis methodologies. These include a combination of qualitative and quantitative techniques as explained in detail in a separate section below under the Data Collection and Data Analysis sections. Two districts - namely Mufindi and Mkuranga - were involved and were chosen because of their location in two different regions that are far apart from each other. Each district generally exhibits some different socioeconomic and epidemiologic characteristics and healthcare facility (HF) infrastructure ownership and distribution. While Mufindi is in the Iringa Region, an area with an unstable and seasonal malaria transmission, Mkuranga is in the Coast (Pwani) region, with stable and perennial malaria transmission. Both districts are typically rural. No specific intervention was conducted in this study to test some hypothesis an compare the results based on the different criteria used to choose the two districts, but the investigation was carried out with desire to find out what the results would show in terms of similarities and differences between the two districts Additional notes on the reasons for their choice are documented elsewhere (Mubyazi et al., 2010; Mubyazi et al., 2014a; Mubyazi et al., 2014b).

The main data sources were pregnant women recruited at $\mathrm{HF}$ level while attending ANC clinics and at village level. The latter group of women were investigated along with the mothers of infants and young children who not older than two years as such women were recent users of ANC services. The term 'current ANC users' as applied throughout the present paper is taken to refer to Women who were currently pregnant on date (of study) while the term 'recent ANC users' refers to women who had given birth within the last twenty four months period prior to the present study. Information from the recent and current ANC users was, supplementary data with informal (personal) communication with frontline HWs who were found at same ANC clinics selected for investigating pregnant women (Mubyazi et al., 2012), as 
well as some intertemporal observations made at the selected clinics (Mubyazi et al., 2014a).

Included in the study were HFs owned by the government (public sector) and those belonging to the private sector, and these were identified in rural and urban settings. In total, $28 \mathrm{HFs}$ were covered to allow selection of the ANC clients for study. Mixed methods were adopted to identify the HFs and these consisted of 13 in Mkuranga and 15 in Mufindi. Several facilities, mostly being dispensaries, were selected using a simple random sampling strategy; others (hospitals and several health centres) were identified using a convenience sampling strategy. More details on the sampling strategy for the HFs are documented elsewhere (Mubyazi et al., 2010; Mubyazi et al., 2014a; Mubyazi et al., 2014b). A convenience sampling approach was also adopted when identifying the current users of the ANC services on particular study days while a purposive sampling method/strategy was used to recruit participants at village level for the group discussions. The pregnant women found at clinic levels accounted for 417 and 406 from Mkuranga and Mufindi, respectively, making a total of 823 ANC clients recruited at HF levels (but as shown later in the data analysis and results sections, some data on 2 questionnaires defaulted and therefore were dropped). Twelve group discussions were held with these women in each district, six groups with pregnant women and another six with mothers of young children (each group was comprised of 6-12 participants). Inclusion of mothers of little children and pregnant women found at village level was targeted to capture feedback from those who might not be using the ANC services during their pregnancy period for various reasons, and their views would be compared with those from the pregnant women who coincidently were found visiting the ANC clinics at the selected study HFs. The HWs involved in the interviews included those who delivered services to ANC clients, and their overall samples and type of cadres have been documented in previous publications (Mubyazi et al., 2012; Mubyazi et al., 2014a).

\section{Data collection}

The data collection for this sub study covered the period March-October 2006. The techniques used included: (i) a questionnaire with standardized (structured) questions administered face-to-face with the ANC clients at HF levels; (ii) focus group discussions (FGDs) with women found in the villages; (iii) informal communication with the HWs; and (iv) researchers' direct inter-temporal observations, focusing on records shown on the pregnant women's ANC cards and other types of information given to clients by HWs. The questionnaire contained mostly closed (precoded questions supplemented with a few open-ended questions). In the FGD, and personal (informal) communication guides, questions addressed the study participants' knowledge of the kind of services they received at the HF levels, perceptions of the quality of services, and implications for actual utilization of IPTp-SP (Mubyazi et al., 2014a). Finally, participants were given a chance to suggest what they believed (if taken seriously as measures) would have enhanced the level of accessibility and utilization of ANC services.

\section{Data processing and analysis}

Quantitative data from questionnaires were mostly categorical. This paper limits its reporting to the tabulated frequency distributions of responses given to specific questions. The number of the responses to specific questions on the questionnaire varied and this limited use of a single and common denominator. This is the reason for the differences or variations in the percentages shown to represent the number of positive answers to each question as a proportion of numbers of interviewees responding to the particular question.

\section{Ethical considerations}

National ethical approval No. NIMR/HQ/R.8a/ Vol. IX/400 for this study was granted by the Medical Research Coordinating Committee. Regional and district central and local government authorities were contacted earlier from the time the overall study proposal was completed before its submission to the national ethical clearance authority. The latter granted study permission in their area of jurisdiction, a copy of which was shared with the respective regional and district authorities. All individual study participants in the study fields were informed of the study objectives, advantages and implementation and dissemination strategies prior to requesting them to sign an informed consent form, and only those who expressed consent to participate and sign were admitted. Participants' freedom to participate or decline participation without coercion or one facing any negative consequences, and issues of anonymity and confidentiality were also observed.

\section{Results}

\section{Characteristics of study participants}

The mean age of the respondents was 23.6 years and the range was 15-46 years in Mkuranga, while in Mufindi, the mean age was 25.0 years, the range being $15-47$ years. Fifty seven percent (238) of the 417 respondents in Mkuranga were standard seven primary school leavers 
with the remaining 179 (42.9\%) having had no formal schooling. In contrast in Mufindi, 357 (89\%) out of 403 respondents were standard seven leavers; the rest had never attended a formal school.

The data showed that in Mkuranga, 239 (57\%) out of 417 interviewees had been pregnant at least once before the present study interviews; the rest of the women were primigravidae. In Mufindi, 327 (81\%) out of 403 interviewees had been pregnant at least once prior to their current pregnancy; the remaining $76(18.9 \%)$ were primigravidae.

\section{Antenatal attendance}

\section{Reasons for ANC visits}

The most common reason given for attending ANC services was to be informed about the development of the fetus and about the pregnancy in general. Fifty six percent of respondents (234 out of 417) in Mkuranga and $284(70.5 \%)$ out of 403 respondents in Mufindi stated the primary reason for their decision to attend clinic to be their desire to receive health education and advice on their pregnancies. Another $23 \%$ of 97 respondents in Mkuranga and $29(7.2 \%)$ of their fellows in Mufindi indicated the desire of being screened of blood and urine for checking illness causes as their main reason for seeking ANC. The next most common reason for one's decision to visit a clinic was the need to be vaccinated for immunizable infectious diseases as indicated by 23 $(5.5 \%)$ and $10(2.5 \%)$ of the respondents in Mkuranga and Mufindi, respectively. Confirming pregnancy status (through a pregnancy test or other means used by the HWs) was stated by $8(1.9 \%)$ respondents in Mkuranga and $18(4.5 \%)$ respondents in Mufindi. Being advised of the gestational age was mentioned by $19(4.6 \%)$ respondents in Mkuranga and $2(0.5 \%)$ in Mufindi. Only $5(1.2 \%)$ respondents in Mkuranga and $19(4.7 \%)$ in Mufindi identified the desire to receive IPTp as the main reason for contacting the ANC clinic. Additional reasons but identified by a few were the desire to receive price discounted insecticide treated net vouchers; an attempt to respond to pressure from family or community members if one seemed unprepared to attend clinic and therefore avoiding blame from either family members or the community or HWs if anything went wrong should one not attend the clinic.

\section{Reasons for not attending ANC clinic and perceived risks}

The reasons stated include one's late recognition of pregnancy status, b) unwanted pregnancy and stigmatisation by either the relatives or the community at large or by HWs; domestic (including family and social) or official occupational commitments, and perceived lack of benefits of attending clinic. Participants in the
FGDs in both districts claimed that late recognition of one's pregnancy condition was mainly experienced by young pregnant women, especially primigravidae. All participants in both districts acknowledged such late pregnancy recognition as having contributed to one's late registration for ANC services and therefore making some women to miss IPTp in the recommended period. As claimed, this problem was more commonly experienced when the woman concerned did not have any close relative in the community in which she lived to help her detect or recognize the early symptoms and signs of pregnancy and who could immediately provide the necessary advice on pregnancy care. In both districts' FGD sessions, participants acknowledged the national school health program for helping to identify pregnant teens or adolescents and direct them to register for ANC clinic services immediately. Late recognition of pregnancy was also reported to be associated with mothers conceiving while lactating or the women who continue facing menstruating after conceiving. Even when an assumed pregnancy is recognised early and the clinic is approached, failure by some clinic HWs to confirm pregnancy was reported as common in both study districts. The women concerned were forced to accept advice from these service providers. As argued, such providers required the respective women to choose either revisiting the same clinic several times in the scheduled weeks or visit any other HF with laboratory services for carrying out a pregnancy test and give reliable results instantly. For fear of being rebuked/ reprimanded or mocked by HWs at other HFs because of contacting different clinics during the pregnancy, it was not uncommon for the latter category of pregnant women to wait until the pregnancy signs were evident for them to revisit the originally contacted clinics/HWs. This late care seeking behaviour was reported to limit the eligible clients to receive IPTp doses and other basic ANC services in the recommended time.

\section{Concern regarding unsatisfactory quality of the ANC services}

During FGDs in both districts, the low opinion of the quality of ANC services was mainly related to the possibility of being exposed to negative staff behaviour, especially the use of discouraging language for the clients coming late to the clinic or those carrying multiple pregnancies with a small child spacing period. At Nyololo village in Mufindi, a FGD member explained this, provoking laughter supporting the claim: "Sometimes pregnant women decide to cheat by stating the incorrect number of pregnancies they have ever carried in order to escape from insults or reprimands by the nurses". A similar experience 
was reported by their counterparts in Mkuranga. In both districts it was argued that multigravidae women seemed not expressing any worry about possible negative pregnancy outcomes while fear was common among those who never experienced such conditions during their past pregnancies. Mishandling pregnant women by some of the lowly qualified or poorly behaving HWs was identified to be a cause of distrust in HWs.

Knowledge about the antenatal services received at the clinics

\section{The package of ANC services}

Table 1 shows the reported information exchange between the service users and HWs at the study clinics on the day when the interviews were conducted. As illustrated, the highest levels of responses (50-80\%) addressed issues related to the general health of the pregnant woman, her pregnancy history including the number of pregnancies/deliveries prior to the current pregnancy, the gestational age of the foetus and foetal movements, as well as the client's current dwelling place. The less frequently $(35-50 \%)$ reported information being sought or provided by the HWs considering the specifications or recommendations given in the national focused ANC guidelines was related to issues of the use of contraceptives, bednets, risks/dangers of tobacco and alcohol use during pregnancy, need for taking one's history of recent vaccinations, intake of antimalarial drugs, and referral preferences. Though infrequently mentioned (by $<35 \%$ of the interviewed ANC clients), the issue of one's cash income and a client being allowed to ask questions (if any) were raised with concern.

Table 1. Information exchange confirmed by respondents in Mkuranga ( $N=417)$ and Mufindi $(\mathrm{N}=403)$ districts to have taken place at ANC clinics on the day of the interview

\begin{tabular}{|c|c|c|}
\hline Questions about the provision of antenatal care services & Mkuranga & Mufindi \\
\hline Have you been informed about the services you were going to be offered? & $150(36.1 \%)$ & $65(16.1 \%)$ \\
\hline Have you been asked about your health condition in general? & $224(53.9 \%)$ & $199(49.4 \%)$ \\
\hline Have you been asked to present your pregnancy history? & $178(53.9 \%)$ & $267(66.3 \%)$ \\
\hline Have you been asked about the anticipated gestational age of your pregnancy? & $267(64.2 \%)$ & $306(75.9 \%)$ \\
\hline Have you been asked about experiencing foetal movement in the womb? & $307(73.8 \%)$ & $313(77.7 \%)$ \\
\hline Have you been asked if you have ever used contraceptives before? & $76(18.3 \%)$ & $178(44.2 \%)$ \\
\hline Have you been asked whether you have ever used any drugs before and how? & $62(14.9 \%)$ & $134(33.3 \%)$ \\
\hline Have you been asked if you use tobacco or alcohol during your pregnancy? & $24(5.8 \%)$ & $145(35.6 \%)$ \\
\hline $\begin{array}{l}\text { 9. Have you been asked if you have experienced sexually transmitted illnesses during your } \\
\text { pregnancy? }\end{array}$ & $57(13.7 \%)$ & $148(36.7 \%)$ \\
\hline 10. Have you been asked whether you sleep under an ITN? & $125(30.1 \%)$ & $185(45.9 \%)$ \\
\hline 11. Have you been asked if you took any antimalarial drug before coming to this clinic? & $164(39.4 \%)$ & $196(48.6 \%)$ \\
\hline 12. Have you been asked if you have been vaccinated before attending this clinic? & $164(39.4 \%)$ & $196(48.6 \%)$ \\
\hline 13. Have you been asked about where you live? & $210(50.5 \%)$ & $243(60.3 \%)$ \\
\hline 14. Have you been asked about your current personal cash income? & $45(10.8 \%)$ & $135(33.5 \%)$ \\
\hline 15. Have you been asked about where you wished to be referred in case of an emergency? & $37(8.9 \%)$ & $158(39.2 \%)$ \\
\hline 16. Have you been asked whether you have any other problem related to your pregnancy? & $62(14.9 \%)$ & $130(32.3 \%)$ \\
\hline 17. Have you been given a chance to ask any questions to the staff? & $28(6.7 \%)$ & $86(21.3 \%)$ \\
\hline 18. Have you been commended for coming for service to the clinic? & $118(28.4 \%)$ & $130(32.3 \%)$ \\
\hline
\end{tabular}

Table 2 shows the respondents' reports on the extent to which the ANC services, including provision of health information and advice (health education) were provided at HF by HWs on the particular present study day. As shown, more than $50 \%$ of the respondents identified such services as blood pressure measurement, foetal palpation, noting of the foetal movement, advice or health information on personal hygiene, preparations for delivery, immunization services, and nutrition. Services confirmed by $35-50 \%$ of the respondents as having been provided were pulse rate measurement, examination for anaemia, breast and abdominal examination, measurement of haemoglobin levels, advice provided on the importance of resting during pregnancy, and health information provided about danger signs, proper use of medicine, sexually transmitted infections (STI), and IPTp. Finally, the services confirmed as having been provided infrequently ( $<35 \%$ of respondents) were measurement 
of body temperature, examination of genital organs, testing for STI, urine collection and screening, and health information provided about safe breastfeeding, risky behaviour, and possible side effects of SP.

Table 2. Services related to clinical examination confirmed by respondents in Mkuranga $(\mathrm{N}=417)$ and Mufindi (N=403) districts to have been received at ANC clinics on the day of the interview

\begin{tabular}{|c|c|c|}
\hline Acknowledgement on types of services received at the clinic & Mkuranga & Mufindi \\
\hline 1. Blood pressure measured & $237(56.9 \%)$ & $247(61.4 \%)$ \\
\hline 2. Body temperature measured & $36(8.7 \%)$ & $124(30.8 \%)$ \\
\hline 3. Pulse measured & $133(31.9 \%)$ & $203(50.4 \%)$ \\
\hline 4. Examined for anaemia & $185(44.5 \%)$ & $184(45.6 \%)$ \\
\hline 5. Breast examination & $62(14.9 \%)$ & $193(47.9 \%)$ \\
\hline Abdomen examined & $149(35.8 \%)$ & $225(55.8 \%)$ \\
\hline 7. Foetal palpation & $353(84.9 \%)$ & $377(93.6 \%)$ \\
\hline 8. Foetal movement noted and information relayed (to pregnant women) & $227(54.6 \%)$ & $271(67.3 \%)$ \\
\hline 9. Genital organs examined & $23(5.5 \%)$ & $105(26.1 \%)$ \\
\hline 10. Testing for sexually transmitted infections (STI) & $40(9.6 \%)$ & $62(15.4 \%)$ \\
\hline 11. Haemoglobin level measured & $194(46.6 \%)$ & $154(38.1 \%)$ \\
\hline 12. Urine collected and screened & $88(21.2 \%)$ & $14(3.5 \%)$ \\
\hline 13. Advice provided about the importance of resting during pregnancy & $47(32.9 \%)$ & $138(60.3 \%)$ \\
\hline 14. Health information provided about personal hygiene during pregnancy & $69(48.3 \%)$ & $135(58.9 \%)$ \\
\hline 15. Health information provided about how to prepare for the delivery & $68(47.6 \%)$ & $152(66.7 \%)$ \\
\hline 16. Health information provided about danger signs during pregnancy & $51(35.7 \%)$ & $136(59.6 \%)$ \\
\hline 17. Health information provided about proper use of medicine during pregnancy & $41(28.7 \%)$ & $128(56.1 \%)$ \\
\hline 18. Health information provided about immunization services & $62(43.9 \%)$ & $144(63.3 \%)$ \\
\hline 19. Health information provided about nutritional issues for pregnant women & $74(51.8 \%)$ & $132(57.6 \%)$ \\
\hline 20. Health information provided about sexually transmitted infections (STI) & $46(32.6 \%)$ & $117(51.3 \%)$ \\
\hline 21. Health information provided about safe breastfeeding & $13(9.2 \%)$ & $88(38.6 \%)$ \\
\hline 22. Health information provided about risky behaviour during pregnancy & $28(19.7 \%)$ & $96(42.1 \%)$ \\
\hline 23. Health information provided about IPTp services & $55(39.0 \%)$ & $136(59.7 \%)$ \\
\hline 24. Health information provided about possible discomfort after taking SP for IPTp & $9(6.4 \%)$ & $33(14.5 \%)$ \\
\hline
\end{tabular}

\section{Knowledge of IPTp as part of ANC services}

Two hundred and forty-four (58.7\%) out of 416 respondents to the question in Mkuranga and $317(80.5 \%)$ out of 394 respondents in Mufindi confirmed that they had received anti-malarial drugs. In contrast, $28(6.7 \%)$ and $10(2.5 \%)$ in Mkuranga and Mufindi, respectively denied having been provided this service while the rest were not sure.

With respect to the name of the anti-malarial drug being provided to eligible pregnant women, $174(68.0 \%)$ out of 256 respondents in Mkuranga and 176 (54.3\%) out of 324 respondents in Mufindi correctly identified SP, Orodar or Fansidar. In Mkuranga, 123 (29.6\%) out of 416 respondents acknowledged they had received SP on the day of the interview while $229(55.0 \%)$ had not; the rest could either not remember or be sure of whether they had received it or not. In Mufindi, 179 (45.4\%) out of 394 respondents acknowledged receiving SP on the day of interview, but 137 (34.8\%) denied having received it; the rest were unsure if they received or not. The respondents confirming to have received SP on the day of the interview and taken it under direct observation by a HW accounted for $59(47.9 \%)$ and $152(84.9 \%)$ in Mkuranga and Mufindi, respectively.

Participants who confirmed having been given SP for IPTp and allowed to take it unsupervised at home were asked to show the tablets provided. Some of them showed paracetamol or Panadol or ferrous/folic acid tables instead of SP. According to the sermons from the HWs interviewed, allowing the clients to take SP at home was done on belief that the respective clients would comply with the instructions. So, it came to be discovered later that some of the clients after leaving the clinic threw the tablets away or kept the tablets with them until next time they revisit the clinic. Evidently, several clients who were approached by the present study interviewers were found with plastic bags containing medicinal tablets among which was the SP given either on that particular day or sometimes before during the last ANC visit made by the respective clients. In Mkuranga, 54 respondents 
$(43.9 \%)$ out of 123 who reporting to have received SP on the study day showed the real SP tablets, but $18(14.8 \%)$ presented other types of tablets. In Mufindi, 20 (11.2\%) out of those who affirmed to have received SP for IPTp showed the real SP tablets while $36(20 \%)$ showed other types of drugs including mebendazole, iron and vitamin tablets. The rest of the respondents did not show any drug.

During FGDs in Mufindi, it transpired that SP and Fansidar were often believed as different drugs. Some of the participants during both exit interviews at HF level and FGDs at community level went further by claiming Fansidar as a better medicine than SP. The betterment of Fansidar was perceived in terms of its ability to yield better treatment outcomes such as not physically exhausting the person swallowing it and working faster in the body to bring relief to its user. Similarly, in Mkuranga, SP and Metakelfin were typically considered to be two different types of drugs while several others believed SP and Panadol to be the same because of their structural appearance. The view that SP was provided as a malaria preventative measure was expressed by 236 (88.4\%) out of 267 respondents in Mkuranga and by 217 (86.1\%) out of 252 respondents in Mufindi, but the difference in the response rates between the two districts was not statistically significant.

From FGDs conducted in both districts, it transpired that the concept of IPTp was yet to be well understood to many community members who commonly perceived $\mathrm{SP}$ to be suitable and delivered for treating confirmed malaria and not a presumptive treatment. However few they were, several FGD members expressed reservations about taking SP at the clinic without clinical or laboratory examination. They believed that lab test or a proper clinical observation would help to confirm malaria and provide reliable results that would guide the service provider to prescribe medicine. Giving one SP tablets to swallow on the spot at the clinic in front of the HW before either of the latter two confirmatory malaria tests confused the clients who regularly heard adverts from government health authorities or private pharmaceutical companies warning the general public and patients of the habit of self-medication or drug taking without undergoing proper medical checkups.

From the overall data of 821 interviewees in both study districts combined, $561(68.3 \%)$ stated to be aware of pregnant women receiving IPTp at ANC clinics. Of those aware, $97(17.3 \%)$ were primigravidae and 464 $(82.7 \%)$ were multigravidae. Of the respondents who acknowledged to have had been informed of the IPTpSP strategy as practically being implemented in HFs, 441
(78.6\%) were found at public HFs whereas 120 (21.4\%) at private clinics. Specifically, $173(70.0 \%)$ out of 247 respondents selected at public HFs and $71(42.0 \%)$ out of 169 respondents selected at private HFs in Mkuranga acknowledged IPTp doses to have been provided; the rest in each case were unaware. In the Mufindi district, $268(97.1 \%)$ out of 276 respondents among those found at public HFs and $49(96.1 \%)$ of 51 respondents among those found at private HFs acknowledged being aware ofthe IPTp service.

The national ANC policy guidelines require each pregnant woman seeking ANC to be given an antenatal card where her pregnancy history and the services delivered to her for each Antenatal visit should be recorded (MoHSW 2004) as should be done in the health management information system (MTUHA) registers (Mubyazi et al., 2014b). Intertemporal observation on the card of 417 interviewees in Mkuranga and 404 cards of their counterparts in Mufindi showed that 210 (50.4\%) and 199 $(49.3 \%)$ cards in these districts, respectively, belonged to clients who had so far received only the first dose (IPTp$1)$, respectively. Those having received the second dose (IPTp-2) accounted for 110 (52.4\%) and 152 (76.4\%) in the latter two districts, respectively. However, 33 (30\%) respondents in Mkuranga and 68 (44.7\%) in Mufindi also had received the third dose (IPTp-3).

\section{Perceived influence of HF structures, staffing levels and other resources on ANC seeking}

Interestingly, the respondents from both districts recognized the influence of $\mathrm{HF}$ structural conditions on pregnant women's antenatal attendances. In regard to existing facilities and available material and human resources at the ANC clinics, $152(36.5 \%)$ out of 417 respondents in Mkuranga and 143 (35.5\%) out of 403 respondents in Mufindi thought the staffing levels were adequate. In contrast, $220(52.8 \%)$ and $233(57.8 \%)$ in these districts, respectively found the staffing levels to be seriously inadequate and compromising the quality of the service. The waiting time at the ANC clinic was regarded as acceptable by $136(32.6 \%)$ respondents in Mkuranga and by 158 (39.2\%) in Mufindi, whereas $202(48.4 \%)$ and $170(42.2 \%)$ in these districts, respectively, found them unacceptably long. The condition of the consultation rooms at the ANC clinic was viewed as satisfactory by 215 (51.6\%) respondents in Mkuranga and by 239 (59.3\%) in Mufindi, whereas $82(19.7 \%)$ respondents in Mkuranga and $48(11.9 \%)$ in Mufindi found them unsatisfactory. In $53(12.7 \%)$ and $76(18.9 \%)$ of the respondents in Mkuranga and Mufindi, respectively, claimed that the HF consultation rooms were either too few or did not exist 
at all at their clinic. Examination beds were perceived to be satisfactory by $252(60.4 \%)$ and $261(64.8 \%)$ of the respondents in Mkuranga and Mufindi, respectively. In contrast, $90(21.6 \%)$ and $85(21.1 \%)$ of the respondents in these districts, respectively, found them unsatisfactory. Fifty-two $(12.5 \%)$ and $45(11.2 \%)$ of respondents in Mkuranga and Mufindi, respectively, perceived the examination beds to be fewer than required and almost non-existing. While toilets at the clinic were found to be in acceptable use/condition by 113 (27.1\%) and 158 (39.2\%) of the respondents in Mkuranga and Mufindi, respectively, $86(20.6 \%)$ and $57(14.1 \%)$ these districts, respectively were not happy. The poor conditions identified were reported a disappointment to service users and hinder some of them to visit HFs.

Likewise, from the FGDs conducted at community level it was remarked that most peripheral government dispensaries lacked separate rooms in the $\mathrm{MCH}$ unit for delivering more sensitive services such as those required for maintaining patient privacy. As also confirmed by frontline HWs, pregnant women, especially those already with multiple pregnancies, were feeling humiliated when attended by a HW in an open area and were being advised on family planning or HIV/AIDS prevention and treatment matters in the presence of other clients (including young mothers).

Through direct observations the team involved in the present study confirmed the claims about inadequate staffing levels and HF infrastructure and equipment shortages. Space meagreness forced a considerable number of the clients to wait outside of the building, facing overheating from the sun or rain. Unquantified information from both the interviewees and FGD participants supplemented by observations indicated an indirect influence of such a service environment on the motivation of some of the clients to visit the clinic frequently.

\section{Suggested policy and management options for improving quality of ANC}

When asked about the perceived need for improving ANC services, 57 (13.7\%) out of 417 respondents in Mkuranga and $103(25.5 \%)$ out of 404 respondents in Mufindi indicated that all services needed some kind of improvement. The views given by individual interviewees and FGD participants regarding the potential policy or management options needed for improving and sustaining delivery of quality services are as documented. They suggested removal of user fees for ANC, strengthening safe motherhood education, provision of bednets for free to all ANC clients, improve health infrastructure facilities used by clients, ensuring availability of essential medicines all the times, increasing number of staff to save waiting time at the HFs, and fixing HFs closer to users to avoid inconvenience and cost of walking long distances.

Opinions were also given regarding the urgent need for the authorities concerned to reconsider cost-sharing for the ambulance service and cost-sharing relating to women's contribution to the procurement of essential delivery materials. When informed that the discounted insecticide-treated net vouchers currently offered to all pregnant women and mothers with children under five years of age who attend $\mathrm{MCH}$ clinics were meant to motivate the eligible clients to access them by attending clinic, most of the view givers in Mkuranga challenged that by claiming that after all the advocated nets were actually not accessible. Accessibility was defined in terms of the distances people were forced to travel to reach the accredited net stores/shops since the poor women who did not receive any monetary support from their spouses or other close relatives found this unaffordable to them. In Mufindi, the same concern was expressed to a lesser degree. Onion givers suggested increased accessibility to HFs, laboratory services, ITNs and drugs by making such facilities and products available closer to the target users was also proposed, although this should go hand in hand with increasing the number and skills of staff periodically posted to various peripheral HFs for them to work with higher motivation and better attitude toward clients.

\section{Discussion}

ANC-seeking behaviour influenced by knowledge of the services being delivered

The findings from the present study reveal that the prime motivation for women registering early at the clinic was one's eagerness to know the progress of their pregnancy in terms of safety and foetal growth rather than knowing the possible risks to the foetus and its mother including exposure to malaria morbidity and mortality episodes. Some of the study present results indicate low percentages/number of the responses or the respondents to particular questions when specific elements of ANC services were mentioned. However, it might not be correct to judge that the unknown services have not been actually delivered or were not considered a priority by the individual ANC respondents. As evidence from studies conducted all over sub-Saharan Africa shows, pregnant women may know and appreciate the benefits of $\mathrm{MCH}$ services (e.g. ANC including IPTp-SP) and the need for seeking such services regularly as recommended, but 
coming to be hindered by contextual (including socialcultural, economic and even systemic) factors to comply with the care seeking schedules/appointments (Mubyazi et al., 2014a; Pell et al., 2011). Various contextual factors including social-cultural barriers such as social stigma against unwanted pregnancies and negative attitudes shown by frontline HWs to clients contribute delaying or hindering target service users to initiate visits to clinics or maintain their attendance according to the scheduled visits.

\section{Women's knowledge of the ANC services actually delivered on the date of interview}

The descriptive statistical data (Table $1 \& 2$ ) may show that some of the basic ANC services have been actually provided. It is possible that some of the services have actually been delivered but the clients concerned did not recognize them. Similarly, some of the services reported to have been provided had not actually been delivered. The observed lack of knowledge among some of the respondents with interview schedules about whether or not SP was given on the study day confirm the latter argument. Consistently, another study in eastern Tanzania found a considerable proportion of pregnant women being unaware of IPTp, including its uptake and the timing of the correct doses, despite attending ANC clinics (Brieger 2008). Therefore, answers collected based on opinions given by respondents through word of mouth without other mechanisms being used to confirm them need to be treated cautiously (Asenso-Okyere et al., 1999). However, these latter findings/evidence are validated by reports from the frontline HWs in the present study who confessed to have occasionally found themselves too busy with heavy workload at HFs to deliver the required health messages or keep individual clients' records for the services delivered in the appropriate official registers or personal HF cards (Mubyazi et al., 2012; Mubyazi et al., 2014a).

Implications for the proposed measures for improving delivery and utilization of ANC services on IPTp-SP uptake and coverage

It is interesting to see how the suggestions given by the study participants in both FGDs and exit interviews in relation to need for waiving service delivery fees, improving service delivery supplies, human resources and physical conditions of the service delivery environments were linked with the chances for pregnant women to attend ANC clinic and consume IPTp-SP doses. This is important to think about by the authorities concerned with quality of care improvement or maintenance as one can now see how service clients consider quality of care in a multidimensional way - physical, input, process, and outcome quality dimensions. It should not always be taken for granted that clients who are lay persons normally are driven by unwarrantable allegations or beliefs rather than true facts when they express opinions regarding health care quality. Some of the suggestions could be managed or handled through careful planning at district level with the necessary support from higher (e.g. central government authority) levels. By suggesting several possibilities, the present study participants rightly noted that there was not a single measure that could remain as a stand-alone for adoption toward an optimal and effective improvement of $\mathrm{MCH}$ services. The interdependence of some of the elements or factors identified by the respondents may not appear as obvious to everybody, but simple economics shows the possibility, for example of user fee implementation at HF level for specific services or general services deterring service use, at least by the poorest segments of the community, and particularly in relation to IPTp (Mubyazi et al., 2010; Ribera et al., 2007; Worrall et al., 2007).

\section{Study limitations}

Studies on knowledge, attitudes and practices (KAP) assessment or appraisals have limitations and their misuse for explaining health behavior is known. There are issues of reported knowledge that may deviate from biomedical/scientific knowledge; people tend to respond to questions assessing their attitudes and practices intelligently. There is tendency to tell what may please or surprise the investigator while the information giver knows that the information given is either false/ wrong or unreliable. Thus, KAP studies are often largely descriptive in nature and over rely on verbal explanations of the person who responds to the question(s). It is not uncommon to find that part of the information given is speculative or biased (Hausmann-Muela et al., 2003). The study findings represent experiences and views of the sample of women selected at HFs and community settings in only two districts. After all, those interviewed at HF levels might have shied away from discussing certain issues openly and widely. Moreover, data analysis was based on a small amount of the data on certain aspects from subsamples that may not be representative of the entire rural community of the respective study regions or the country as a whole. The proportions/ frequencies of some categorical responses are too small while missing values for several questions had been dropped off; hence limiting conduction of a detailed analysis that would provide for a comparative assessment of the responses obtained from the women groups with different socioeconomic characteristics and from HFs 
belonging to different service providers. Finally, the cross-sectional nature of the study is another criticism since the KAP of the person may change over time and therefore can better be assessed through longitudinal (cohort-designed) studies.

\section{Conclusions}

This study suggests that generally ANC care involving IPTp administration is positively perceived by the majority of pregnant women in principle. This is because the respective women seem to consider malaria as a risky disease among other diseases affecting pregnant women. The suggestions given by the study participants imply that any target or attempt to promote IPTp usage and coverage should be done by ensuring improvement in the delivery of $\mathrm{MCH}$ in general. A client may need to undergo health education, voluntary counselling and later testing before being prescribed medicines, but these services should be provided in acceptable HF and these are ones adequately equipped with skilled and motivated staff as well as essential medicines and other materials. Whether the service clients were correct or not in their allegations against frontline HWs or concerns about the missing services is not an important issue/question, instead, the authorities concerned need to appreciate that service clients are best persons to tell what they feel about the services and as helpful agents of change through their constructive criticisms in line with patient charters (Creel et al., 2002; van Duong et al., 2004; Rao et al., 2006). That is why there is need for periodic evaluations of population's knowledge and perceptions of the existing national policy and its accompanied interventions as this would help to allow the authorities concerned take all the necessary initiatives or corrective actions.

\section{Conflict of interest}

The author declares no conflict of interest in this paper.

\section{Author's contributions}

The present author, GMM, conceived the study, and implemented it as its principal investigator as part of his $\mathrm{PhD}$ training requirements at the University of Copenhagen in Denmark. GMM wrote the manuscript.

\section{Acknowledgment}

The Bill and Melinda Gates Foundation through the Gates Malaria Partnership (GMP) coordinated by the London School of Hygiene and Tropical Medicine (LSHTM) provided funding for this study. Thanks to the GMP Director (Prof. Brian Greenwood) on behalf of GMP staff and management at large and the DBL
Centre for Health Research and Development and Centre for Malaria Parasitology (CMP) at the University of Copenhagen, Denmark as collaborating institutions in this study. The enumerators and administrative assistants for this study and the cooperation received from the Local and Central Government Authorities in the study regions are invaluable. Professors Ib C. Bygbjerg (ICB), Pascal Magnussen, and Drs. Kristian S. Hansen, Jens Byskov, Oystein E. Olsen and Paul Bloch were co-supervisors of GMM's $\mathrm{PhD}$ training; ICB and $\mathrm{PB}$ were co-lead supervisors. The Director General of the National Institute for Medical Research (NIMR) granted GMM a paid $\mathrm{PhD}$ study leave and permission to publish this study.

\section{References}

Asenso-Okyere WK, Akoto IO, Anum A, Adukonu A. The behaviour of health workers in an era of cost sharing: Ghana's cash and carry system. Trop. Med. Int. Health. 1999; 4(8): 586-593.

Brieger W. Attending antenatal care does not guarantee IPTp uptake. Malaria Matters. 2008 [cited 2008 June $\left.17^{\text {th }}\right]$. Accessed from:http://www.malariafreefuture.org/blog/?cat=3

Charkraborty N, Islam MA, Chowdhury RI, Bari W, Akhter HH. Determinants of the use of maternal health services in rural Bangladesh. Health Promotion International. 2003; 18(4):327-337

Creel LC, Sass JV, Yinger NV. Client-centered quality: clients' perspectives and barriers of receiving Care. New Perspectives on Quality Care. 2002; Volume 2, page 1-8. Accessed from: http//www.prb.org/pdf/NewperspQOC-Client.pdf

Hausmann-Muela S, Ribera JM, Nyamongo I. Health-seeking behavior and the health system response. A paper was commissioned by the LSHTM to provide evidence on important crosscutting issues for the Disease Control Priorities Project, LSHTM, UK.2003.

Maputle MS, Lebese RT, Khoza LB, Shilubane NH, Netshikweta LM. Knowledge and attitudes of pregnant women towards antenatal care services at Tshino Village, Vhembe district, South Africa. African Journal for Physical Health Education, Recreation and Dance. 2013; Vol. 19 (Suppl. 1): 126-137Mathole T, Lindmark G, Majoko F, Ahlberg BM. A qualitative study of women's perspective of antenatal care in a rural area of Zimbabwe. Midwifery. 2004; 20(2):122-132.

Ministry of Health - Tanzania: Focused antenatal care malaria and syphilis in pregnancy: orientation package for service providers. Ministry of Health-Reproductive and Child Health Unit \& NMCP, Dar es Salaam, 2004.

Mubyazi GM, Magnussen P, Goodman C, Bygbjerg IC, Kitua AY, Olsen ØE, Byskov J, Hansen KS. Implementing intermittent preventive treatment for malaria during pregnancy: a review of prospects, achievements, challenges and agenda for research. Open Journal of Tropical Medicine. 2008; 1:92-100

Mubyazi GM. Economic and Other Contextual Determinants 
of the acceptability and practicability of Intermittent Preventive Treatment of Malaria during Pregnancy (IPTp) Strategy in Tanzania. PhD Thesis, University of Copenhagen, Denmark, 2010.

Mubyazi G, Bloch P, Kamugisha M, Kitua A, Ijumba J. Intermittent preventive treatment of malaria during pregnancy in Tanzania: a qualitative study of knowledge, attitudes and practices of district health managers, antenatal care staff and pregnant women in Korogwe district, Northeastern Tanzania. Malar J. 2005; 4:31.

Mubyazi GM, Bloch P, Magnussen P, Olsen ǾE, Byskov J, Hansen KS, Bygbjerg IC. Women's experiences and views about costs of seeking malaria chemoprevention and other antenatal services: a qualitative study from two districts in rural Tanzania. BMC Malaria Journal. 2010; 9:54.

Mubyazi GM, Bloch P. Psychosocial, behavioral and health systems barriers to delivery and uptake of intermittent preventive treatment of malaria in pregnant women in Tanzania - viewpoints of service providers in Mkuranga and Mufindi Districts. BMC Health Services Research. 2014a; 14:15.

Mubyazi GM, Byskov J, Ijumba JN, Magnussen P, Marero M, Mboera LEG, Molteni F, Bloch P. Health facility-based data on women receiving sulphadoxine-pyrimethamine during pregnancy in Tanzania: lessons to learn from a cross-sectional survey in Mkuranga and Mufindi districts and other national survey reports. BMC Reproductive Health. 2014b; 11:6.

Mubyazi GM, Bloch P, Magnussen P, Byskov J, Bygbjerg IC. Supply-related drivers of staff motivation for providing intermittent preventive treatment of malaria during pregnancy in Tanzania: evidence from two rural districts. BMC Malaria Journal. 2012; 11:48.
Mubyazi GM, Hutton G. Rhetoric and Reality of Community Participation in Health Planning, Resource Allocation and Service Delivery: a Review of Reviews, Primary Publications and Grey Literature. Rwanda J .Health Sciences. 2002; 1(1):5165.

National Collaborating Centre for Women's and Children's Health: Antenatal Care: routine for the healthy pregnant women. RCOG Press, UK 2008.

Nganda R. Knowledge of malaria influences use of insecticide treated nets but not intermittent presumptive treatment by pregnant women in Tanzania. Malar J . 2004; 3:42.

Pell C, Straus L, Andrew EVW, Meñaca A, Pool R. Social and Cultural Factors Affecting Uptake of Interventions for Malaria in Pregnancy in Africa: A Systematic Review of the Qualitative Research. PLoS ONE. 2011; 6(7):e22452. doi:10.1371/journal.pone.0022452

Rao M, Clarke A, Samderson C, Hammersley R: Patients' assessment of quality of primary health care compared with objective records based measures of technical quality of care: a cross-sectional study. BMJ. 2006; 333:19

Ribera MJ, Hausmann-Muela S, D’Alessandro U, Grietens PK. Malaria in pregnancy: what can social sciences contribute? PLoS Medicine. 2007; 4(4):e92

van Duong D, Binns CW, Lee AH, Hipgrave DB. Measuring client-perceived quality of maternal services in rural Vietnam. Int. J. Qual Health Care. 2004; 16(6):447-452

World Health Organization (WHO). Malaria Policy Advisory Committee: to the WHO: conclusions and recommendations of March 2013 meeting. Malaria Journal. 2013; 12:213.

Worrall E, Morel C, Shunmay Y, Borghi J, Hill J, Wiseman V, Mills A: The economics of malaria in pregnancy - a review of the evidence and research priorities. Lancet Infectious Diseases. 2007; 7:156-168 